\title{
Patterns, Mathematics, Early Literacy, and Executive Functions
}

\author{
Julie K. Kidd*, Hao Lyu, Matthew S. Peterson, Mehreen Z. Hassan, Debbie A. Gallington, \\ Lauren I. Strauss, Allyson B. Patterson, Robert Pasnak*
}

George Mason University, Fairfax, VA, USA

Email: ${ }^{\star} J k i d d @ g m u . e d u,{ }^{\star}$ rpasnak@gmu.edu

How to cite this paper: Kidd, J. K., Lyu, H., Peterson, M. S., Hassan, M. Z., Gallington, D. A., Strauss, L. I., Patterson, A. B., \& Pasnak, R. (2019). Patterns, Mathematics, Early Literacy, and Executive Functions. Creative Education, 10, 3444-3468. https://doi.org/10.4236/ce.2019.1013266

Received: November 27, 2019

Accepted: December 20, 2019

Published: December 23, 2019

Copyright $\odot 2019$ by author(s) and Scientific Research Publishing Inc. This work is licensed under the Creative Commons Attribution International License (CC BY 4.0). http://creativecommons.org/licenses/by/4.0/

\begin{abstract}
In the fall, kindergartners from 21 classrooms in four urban schools were randomly formed into trios and taught either mathematics or early literacy or social studies, or six types of complex patterns. Measures of mathematics, early literacy, executive functions (EF), and understanding of complex patterns were collected in late spring. Exploratory factor analysis indicated that the early literacy measures, one measure of inhibition, and both mathematics measures comprised one factor. Another inhibition measure, cognitive flexibility, working memory, patterning, and the computation mathematics measure comprised another factor. The correlations obtained and the factor analysis distinguish the cognitive and achievement measures and show the relations between them.
\end{abstract}

\section{Keywords}

Patterning, Early Literacy, Mathematics, Executive Functions

\section{Introduction}

\subsection{What Is a Pattern?}

Patterns are a pervasive and important, but understudied, component of early mathematical knowledge (Rittle-Johnson, Fyfe, Loehr, \& Miller, 2015). In the context of early education, Papic, Mulligan, and Mitchelmore (2011) defined patterns as "replicable regularity," and Bjorklund and Pramling (2014) described patterns as "regularity and sequencing in ordering activities." Mulligan and Mitchelmore (2009) defined a mathematical pattern as "predictable regularity, usually involving numerical, spatial or logical relationships." Sarama and Clements (2009a) used the commonly accepted term "patterning" to describe child- 
ren's understanding of patterns and suggested that "patterning is the search for mathematical regularities and structures, to bring order, cohesion, and predictability to seemingly unorganized situations and facilitate generalizations beyond the information directly available." Patterning is an essential cognitive ability in early mathematics, which includes the ability to identify and describe attributes of objects and similarities and differences between them (Papic, 2007). Mathematics curricula in the early years emphasize the importance of patterns as a foundation for "algebraic" thinking (McGarvey, 2012). Curricular expectations in Pre-K to Grade 2 are typically for children to "recognize," "identify," "duplicate," and "extend" repeating and growing patterns (National Council of Teachers of Mathematics, 2000; National Council of Teachers of Mathematics, 2006).

Papic (2007) described two common types of patterns: repeating patterns and growing patterns. Repeating patterns are the type generally used in patterning instruction. In recent studies, Kidd et al. (2013), Kidd et al. (2014) and Pasnak et al. (2015) introduced more complex patterns, including symmetrical patterns, progressive patterns (involving increasing numbers of elements, sizes or values), rotation patterns, and random repeating patterns.

Bjorklund and Pramling (2014) argue that discerning patterns requires awareness of demarcated units and regularity in how the patterns are developing, what aspects should be accounted for, and what aspects are irrelevant to a specific pattern. Children are usually instructed to identify what a pattern is, but there tend to be few instructions on what a pattern is not (McGarvey, 2012). If children are expected to recognize or identify patterns, what criteria do they use to distinguish patterns from non-patterns? In addition, what criteria do teachers apply to identify a pattern? McGarvey's (2012) research indicated that both young children and teachers used "repetition" and "predictability" as their main criteria, and these criteria were used in various sometimes contradictory ways.

\subsection{Patterning Instruction and Its Effects on Academic Achievements}

Recent research shows patterning instruction (i.e., patterning) improves students' mathematical achievements in terms of facilitating their "algebraic" thinking (e.g., Ferrara \& Sinclair, 2016; Papic, 2013; Papic, 2015; Warren \& Cooper, 2008) and predicting later mathematical skills and achievements (e.g., Fyfe, Evans, Matz, Hunt, \& Alibali, 2017; Mulligan, 2011; Mulligan \& Mitchelmore, 2009; Nguyen et al., 2016; Papic et al., 2011; Rittle-Johnson, Fyfe, Hofer, \& Farran, 2016; Warren \& Miller, 2013).

\subsection{Empirical Evidence of Relations between Understanding Patterns and Achievement}

Fyfe et al. (2017) evaluated the relationship between patterning and specific aspects of mathematics in early childhood. They had children aged from 5 to 13 years old complete a patterning extension task with three types of patterns in various levels of difficulty. In addition, they tested the children's calculation 
skills and knowledge of math concepts in equivalence and inversion. Achievement on the patterning extension task was related to mathematics performance. Fyfe et al. (2017) found that patterning ability predicted calculation skills when controlling for age and verbal working memory, but there was no relationship between patterning and knowledge of mathematics concepts.

Mulligan and Mitchelmore (2009) argued that structural development of young children's mathematical thinking is critical to young children's mathematical competence. An interview-based assessment which incorporated various forms of patterns and related features of mathematical and spatial structure, was administered to more than 100 first grade students. The results indicate that awareness of mathematical patterns and structure may be associated with mathematical achievements and it is a construct that could provide new insights into early childhood mathematics learning. Mulligan and Mitchelmore (2009), Mulligan, Mitchelmore, Kemp, Marston, and Highfield (2008), and Mulligan et al. (2017) also reported on a teaching intervention study, explicitly aimed at raising children's awareness of mathematical pattern and structure through various "well-connected pattern-eliciting" experiences. The findings were that the students showed substantial improvement on PASA in terms of growth in identifying subitizing patterns, counting in multiples of two, three, and four, representing, symbolizing and translating simple and complex repetitions, structuring grids and arrays by the end of the project.

Warren and Miller (2013) suggested that there is a clear relationship between one's ability in patterning tasks and one's ability in mathematics. Their study of indigenous Australian students' learning indicated that these children's ability to ascertain the structure of patterns and to understand mathematical language were both strong predictors of their later math achievements.

Rittle-Johnson et al. (2016) also hypothesized that mathematical knowledge begins to develop at a young age and that this early knowledge is critical for children's later academic achievements. Their longitudinal study of over 500 children aged 4 to 11 from low SES families indicated that nonsymbolic quantity, counting, and patterning knowledge in preschool predicted fifth-grade mathematics achievement.

\subsection{Patterning and Cognitive Mechanisms}

Researchers conducting investigations of relations between patterning instruction and academic achievement have called for more basic research on the relationship between patterning and cognitive mechanisms and abilities (e.g., Kidd et al., 2013; Kidd et al., 2014; Pasnak et al., 2015). In the past few years, significant relationships have been found among pattern understanding, executive functions (EF), and relational knowledge (e.g., Bock et al., 2018; Collins \& Laski, 2015; Miller, Rittle-Johnson, Loehr, \& Fyfe, 2016; Rittle-Johnson, Fyfe, McLean, \& McEldoon, 2013; Schmerold et al., 2017). Rittle-Johnson et al. (2013) found that working memory and relational knowledge might be the underlying developmental mechanisms that improve four-year-old's knowledge of repeating 
patterns. Miller et al. (2016) reported that for children aged from four to five years, relational knowledge, working memory, and set shifting (a measure of cognitive flexibility) predicted initial understanding of repeating patterns. In addition, working memory was found to predict improvements in patterning understanding after intervention. Two teams of investigators, Bock et al. (2018) and Schmerold et al. (2017), reported on the relationship between understanding more complex patterns (i.e., symmetrical patterns, different types of growing patterns, and rotation patterns) and executive functioning for six-year-old children. In both cases, even though the measures they used for patterns and EF were different from those Miller et al. (2016) used for preschoolers; their findings were similar, in that patterning was significantly related to two types of EF, cognitive flexibility and working memory, but not to a third, inhibition. The case is not closed, however, for inhibition, because Collins and Laski (2015) found an inhibition effect when they used a more demanding inhibition measure.

\subsection{Implications for Teaching Patterns}

The importance of instruction on patterns has been emphasized in guidelines for early childhood mathematics programs. These include position papers by the National Council of Teacher of Mathematics $(1993,2000,2006)$ and the joint position statement of the National Association for the Education of Young Children/National Council of Teachers of Mathematics Education (2002/2010). Learning about patterns is deemed to help children establish a sound foundation for future mathematics achievements by both these organizations and many researchers (e.g., Clements \& Sarama, 2011; Collins \& Laski, 2015; Dubon \& Shafer, 2010; Fyfe, Rittle-Johnson, \& McNeil, 2015; Kidd et al., 2013; Mulligan, 2011; Papic, 2007; Richardson, Berenson, \& Staley, 2009; Sarama \& Clements, 2009b). However, experimental studies of teaching patterns to children at entry to formal schooling, which in the USA is typically at age five years or very nearly that. Only Mulligan and Mitchelmore's (2009) study of teaching repeating patterns to indigenous Australian children, who averaged nearly six years in age, has involved children close to kindergarten age. Accordingly, the present study was directed at children entering kindergarten.

Although repeating patterns are typically taught to preschoolers, kindergartners might be able to master more complex patterns. By teaching first graders patterns more complicated than repeating patterns Kidd et al. (2013), Kidd et al. (2014), and Pasnak et al. (2015) were able to produce significant gains in both mathematics and early literacy. Hence, we undertook to teach such patterns to kindergartners. One goal was to discover the effects, if any, of this instruction in patterning on kindergartners' mathematics and early literacy. In addition, we hoped to discover what the relations were between these children's understanding of patterns and the major EF, as had previously been done for preschoolers (Collins \& Laski, 2015; Miller et al., 2016; Rittle-Johnson et al., 2013) and first-graders (Bock et al., 2018; Schmerold et al., 2017). 


\section{Method}

\subsection{Participants}

Parental consent was obtained for 204 kindergarten children enrolled in four schools in an urban system in the mid-Atlantic region, which served many families with low incomes and families who are immigrants. Data from the school websites suggests that of these children, 32\% were African American, $45 \%$ were Hispanic, $13 \%$ were Middle Eastern, $4 \%$ were other White, and $6 \%$ were Asian American. A screening test that consisted of patterning, literacy, and math problems was administered, and the 12 children in each of 21 classrooms scoring lowest were selected as the sample for the research. After attrition, 94 girls and 90 boys remained in the research.

\subsection{Research Design and Hypotheses}

The experimental design was that the 12 children in each classroom who scored lowest on patterning, literacy, and mathematics screening tests were matched on the basis of their scores and then randomly assigned to one or another of four instructional conditions. In some classes fewer than eight children returned signed parental permission slips; those who did were matched and randomly assigned to the four conditions. This procedure produced small groups (one to three children in each classroom) who received instruction matched in duration but differing in content. Hence it was an "active" control design (Pasnak \& Howe, 1993: p. 232) wherein all children received equal amounts of attention while engaged in constructive activities.

\subsection{Experimental and Control Conditions}

The experimental condition incorporated a method for teaching patterning that required little skill and was designed to be easily replicated. This instructional approach was intended to teach children to identify six types of complex patterns and fill in the missing part of each pattern. The effects of this experimental instruction were compared to those of three control groups.

Children in the first control condition were taught early literacy, which consisted of instruction in letter and phonics. These children were expected to score better on literacy tests than control children taught mathematics or social studies. The children taught patterning might also score better on literacy measures than those taught mathematics or social studies if the patterning instruction helped them enhance literacy abilities.

Children in the second control condition were taught mathematics by means of activities that were designed to develop children's abilities to recognize, identify, count, and compare numerals. These children should outperform those in the literacy or social studies conditions on mathematics tests. The children taught patterning might also score higher than the literacy and social studies groups on mathematics measures because a better understanding of patterns should result in better mathematics achievement (Economopoulos, 1998; Kidd et 
al., 2013; Kidd et al., 2014; Pasnak et al., 2015). If the patterning and literacy instruction were effective, however, children in the mathematics instructional condition were likely to score lower on patterning and literacy measures.

Children in the third control condition were taught social studies, which primarily involved painting and drawing on worksheets. This instruction was not expected to bring as much improvement in patterning, literacy, or mathematics as intervention directly on those domains. It was designed to provide a baseline for measurement of the effects of patterning instruction and of the two other kinds of control instruction.

\subsection{Measures}

\subsubsection{Screening Tests}

The patterning screening test consisted of a researcher-generated patterning test. The researcher-generated patterning test had 24 six-item sequences of shapes, colors, and objects, presented horizontally in rows on flip charts. The patterns were designed to be presented in specific orders and each pattern equally had one missing item in the beginning or end. Each student was asked to select one item from four alternatives that would fill in the missing spot. There were four types of pattern questions, including $\mathrm{AB}$ patterns (e.g., $\mathrm{ABABAB}$ ), $\mathrm{ABB}$ patterns (e.g., $\mathrm{ABBABB}$ ), random repeating patterns (e.g., $\mathrm{ABCDAB}$ ), and symmetric patterns (e.g., ABCCBA) (see Figure 1).

Students' literacy was assessed with the DIBELS Letter Naming Fluency (LNF) (Marston \& Magnusson, 1988) and DIBELS Initial Sound Fluency (ISF) (Kaminski \& Good, 1996, 1998; Laimon, 1994) scales. The Dynamic Indicators of Basic Early Literacy Skills (DIBELS) are a set of short (one minute) procedures and measures designed to assess the acquisition of early literacy skills. According to Good et al. (2004), for kindergartners and first graders, all DIBELS measures displayed estimated reliability in the $0.90 \mathrm{~s}$ when three or four probes are aggregated together. The median predictive validity of single DIBELS probes with the Woodcock-Johnson Broad Reading Cluster were 0.36 for kindergarten ISF and 0.65 for kindergarten Letters Named Frequency (LNF). By repeating ISF four $(r=0.72)$ to six $(r=0.62)$ times, the resulting aggregate is predicted to have a reliability of 0.91 . For LNF, the median one-month, alternate-form reliability is 0.89 in kindergarten, and the aggregate of two probes has a reliability of 0.94 .

The KeyMath-3 Diagnostic Assessment (Connolly, 2007), a comprehensive, norm-referenced measure of essential mathematical concepts and skills was used for screening students' mathematics knowledge. According to its manual, the internal-consistency reliability of KeyMath-3 is 0.96 for kindergarten through grade 5, and the test-retest reliability of KeyMath-3 Basic Concepts is 0.95 for pre-kindergarten through grade 12. The validity of KeyMath-3 Basic Concepts is 0.97 for kindergarten through grade 2. Convergent reliability of KeyMath-3 is 0.67 - 0.75 with the Kaufman Test of Educational Achievement. 


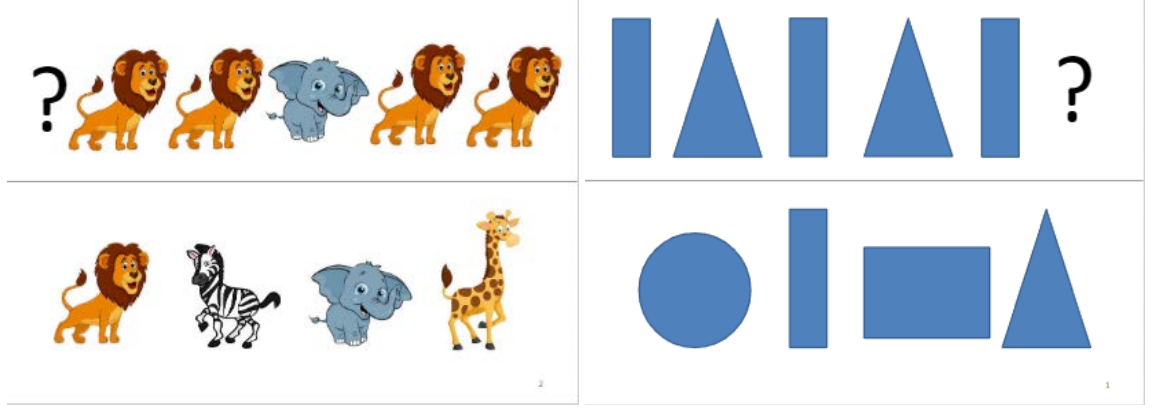

(a)

(b)

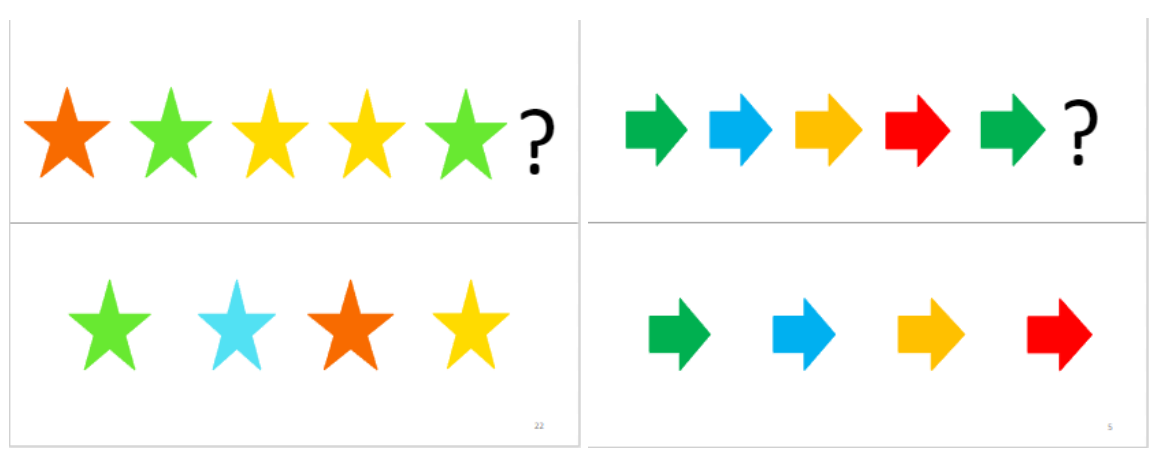

(c)

(d)

Figure 1. Examples of Patterns from the Screening Test. Note. Examples of items and patterns from the patterning screening test. (a) An ABB pattern; objects are presented horizontally, missing the first item. Answer choices are below the line. (b) An AB pattern; shapes are presented horizontally, missing the last item. Answer choices are below the line. (c) A symmetric pattern; colored items are presented horizontally, missing the last item. Answer choices are below the line. (d) A random repeating pattern; colored items are presented horizontally, missing the last item. Answer choices are below the line.

\subsubsection{Posttest Measures}

Posttests were administered in early May of the school year, in individual sessions of no more than 15 minutes. The same patterning test was used for post testing. The DIBELS scales and the two scales of the Test of Word Reading Efficiency (TOWRE; Torgesen, Wagner, \& Rashotte, 2012) were used for posttest measures of early literacy. Wechsler Intelligence Scale for Children-Revised (WISC-R) Digit Span, Multiple Classification Card Sorting Task (MCCST), and Day/Night inhibition test. The TOWRE is designed to assess the fluency and accuracy of students' print-based word-reading and phonemics skills. According to its manual, the TOWRE's reliability ranges from 0.90 to 0.99 . Concurrent validity with the Woodcock Reading Mastery Tests-Revised was from 0.85 to 0.98 .

Two scales from the Kaufman Test of Educational Achievement (KTEA-3; Kaufman \& Kaufman, 2014) were used to assess mastery of mathematics concepts and computations. Split-half reliability ranged from 0.54 to 0.99 (Kaufman \& Kaufman, 2014). Concurrent validity with Wechsler Individual Achievement Tests, Third Edition (WIAT-III; Wechsler, 2009) ranged from 0.18 to 0.95.

The Wechsler Intelligence Scale for Children-Revised (WISC-R; Wechsler, 2014) Digit Span (Backward) was used in this study to assess working memory. 
A sequence of numbers was read aloud to the child, and the child was asked to repeat the numbers in reverse order. The test begins with sequences of only two numbers. The length of each sequence of numbers increased by one digit every two trials as the child responded correctly. For example, the numbers for trial one were " $2-5$ " and for trial two " $6-3$," if the child answered it correctly, the next trials were "5-7-4" and "2-5-9." The test was discontinued when the child responded incorrectly on two consecutive trials.

The Corsi Blocks test was used as another measure of working memory Kessels, van Zandvoort, Postma, Kappelle, \& de Haan (2000). This assessment utilizes nine numbered blocks randomly arranged on a table or board so that only the tester can see the numbers on the blocks. Initially beginning with two blocks, the tester tapped the blocks and the child was instructed to tap blocks in the opposite order. Two trials were given per level, as defined by the number of blocks in the sequence. The task continued until the child failed to correctly copy the order on two consecutive trials on the same level. The score for the task was the largest sequence that the child was able to reproduce.

The Day/Night test is an assessment of inhibition. It was designed to measure an individual's ability to inhibit a prepotent response tendency to perform certain actions. Students were presented 16 pictures of moons and suns in random order. Before starting the test, the researcher turned over a flip chart depicting the moon and instructed the child to say "day" to that flip chart, and then turned over a flip chart depicting the sun and instructed the child to say "night" when shown that flip chart. Both total correct answers and total time the child spent on completing the test were recorded. Chasiotis, Kiessling, Hofer, and Campos (2006) report a high $(r=0.93)$ Kuder-Richardson reliability for the 10 test trials of Day/Night test. Murray, Scott, Connolly, and Wells (2018) report a high ( $r=$ 0.89) Kuder-Richardson reliability for the 20 test trials of Day-Night test.

The Head-Toes-Knees-Shoulder Task (HTKS) is also a measure of inhibition (Ponitz, McClellan, Matthews, \& Morriison, 2009). Children are asked to play a game in which they had to do the opposite of what the tester said. There were two parts to this measure. First, when the tester instructed them to touch their heads, the children were to do the opposite and touch their toes, and vice versa. After 10 trials of part 1, the tester began the second part of the assessment by first coaching the child to touch their shoulders when instructed touch their knees, and vice versa. Ten trials were then administered and included all four instructions (i.e., head, toes, shoulders, and knees). Two points were given to a correct response, 1 for self-corrected, and 0 for incorrect.

The Multiple Classification Card Sorting Task (MCCST) is a measure of cognitive flexibility (Cartwright, 2002). Children were required to sort a set of 12 cards into four piles according to two dimensions simultaneously (i.e., the color and type of object on the card, forming a $2 \times 2$ matrix). Before starting the test, a tester demonstrated sorting a practice set of cards in a $2 \times 2$ matrix to the kids. After answering any questions about the demonstration, a set of 12 cards in 
random order was handed to the child, who was asked to sort them. This was repeated for three other sets of cards, each having different types of objects and colors. Children were scored on the correctness of sorting and the times for sorting each set. After sorting each set, children were asked to explain the reasoning for sorting the cards in the manner they had and this justification was scored on a three-point scale.

\subsubsection{Instructional Materials}

In all instructional conditions, children were provided with small toy ponies or dinosaurs. These props were used to keep the instruction interesting and enjoyable. Questions were always posed to the toy animals, and the child asked to teach his or her animal. This mitigated any negative emotions might result from mistakes.

A variety of researcher-generated instructional patterns were used to teach students sequences, including $\mathrm{AB}$ patterns, $\mathrm{ABB}$ patterns, random repeating patterns, growing patterns, rotation patterns, and symmetrical patterns (see Figure 2). Various elements were used to form each type of patterns, including shapes, colors, objects (e.g., clocks, animals, fruits, etc.), numbers, and letters. The patterns were presented horizontally in rows on flip charts. In addition, whiteboards, foam letters, shapes and numbers, and manipulatives (e.g., big and small colorful beads, cubes, bells, etc.), were used to facilitate the children's patterning learning (see Figure 2).

Children in the mathematics control condition were taught numerals with foam numbers, bingo games, whiteboards, and manipulatives. Foam letters, letter bags (including words and corresponding objects or pictures of objects), note cards (a group of words with the same root, e.g., - air family comprised cards such as chair, flair, hair, lair, pair, and stair), and whiteboards were used to teach children literacy. Play-dough, crayons, and blank pages with various topics (e.g., Abraham Lincoln, toy bear, flower, map of the states, etc.) for painting were used for social studies instruction.

\subsubsection{Procedures}

In early February, during a time where students in each classroom were routinely assigned to receive small group instruction on various topics, the children participating in the research were randomly assigned to predetermined special centers. There they received different forms of instruction-either patterning, literacy, mathematics, or social studies-for 15 minutes a day, three days a week. The types of instruction were given in a counterbalanced order except as interrupted by school events or students' absences. Instruction was directed at each child in turn, while the other two children in that condition watched and then took their turns, either with the same problem or a different one, depending on their levels of ability. At each site, a research manager was assigned to observe the instruction, conduct fidelity checks, and keep records of each child's progress. 


\section{DOPOOPO? 00}

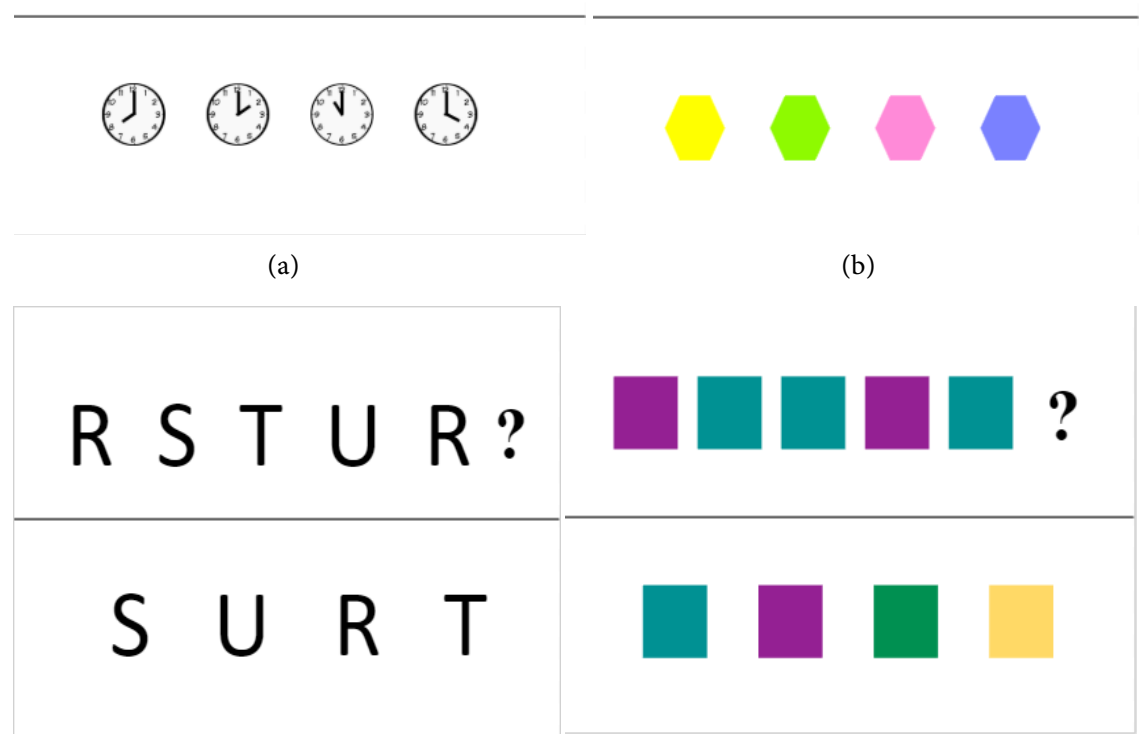

(c)

(d)
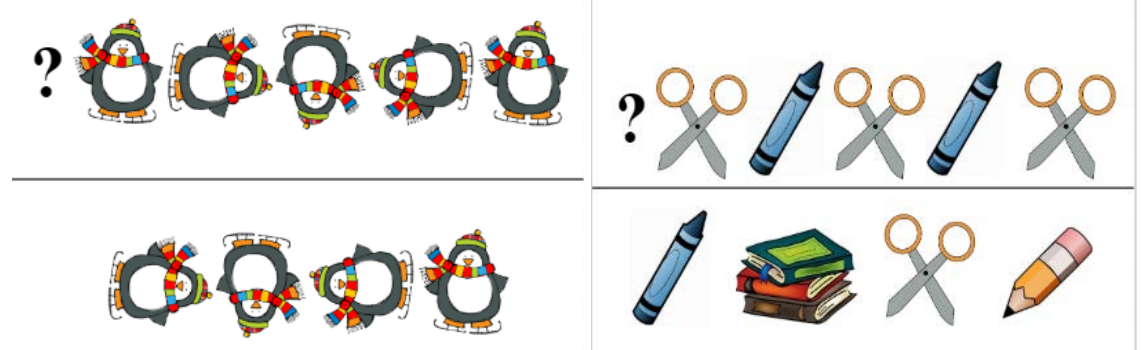

(e)

(f)

Figure 2. Examples of patterns used in instruction. Note. (a) A growing pattern presented with clocks. (b) A symmetrical pattern presented with hexagons. (c) A random repeating pattern presented with uppercase letters. (d) An ABB pattern presented with squares. (e) A rotation pattern presented with penguins. (f) An AB pattern presented with scissors and crayons. Color was used to present the idea of the pattern when it was applicable (e.g., color worked well with shapes to present patterns in example (b) and (d)).

\subsubsection{Procedures in Instruction}

For patterning instruction, the patterns described previously were presented to children one at a time, horizontally in rows on flip charts, while the other two children in that condition watched. Each pattern contained one missing item at the beginning or end of the pattern, and the child was asked to have his or her animal select one of four choices to complete the pattern. Instruction was playful, with each child's animal asked to neigh or growl or give high fives or something else after correct choices. Each flip chart was turned over to the next pattern when the child's animal selected the correct answer, but remained on the 
same page if they picked the wrong answer. Performance was scaffolded through explanation and repetition until each child was able to demonstrate mastery of each pattern type. Each child had a turn during the 15-minute instructional period and might work on the same or different patterns, depending on their level of mastery.

Whiteboards and manipulatives (e.g., beads, bells, cubes, etc.) were also used to enhance each child's ability to identify, create, and extend patterns. A pattern was started and the children provided with manipulatives to complete or extend it. Opportunities were also provided for children to create patterns with manipulatives. Patterns were also drawn on whiteboards were used in a similar way to help children to identify, extend, and create patterns.

Mathematics instruction featured a variety of mathematics-related activities for each day. Foam numbers, bingo games, whiteboards, and manipulatives were utilized in math activities. Specific math concepts or skills, such as numeral recognition, quantifying/counting, number order/spans, and comparisons, were addressed in daily math-related activities. Each mathematics session began with a brief informal assessment to determine the children's mastery of the skill or concept needed in the chosen task. This allowed instruction to begin at appropriate starting points and to be tailored students' abilities. All math activities had fall-back or jump-ahead options which instructors could use to individualize the instruction to meet the needs of the child.

For example, a task that focused on numeral recognition began with asking a child's animal to recognize a numeral (e.g., "Which is number five?") from an ordered array of foam numbers. If the animal failed, it was helped to begin at one and count to the requested numeral, touching and naming each numeral reinforcing both counting and identification. Then the numerals were rearranged in a semi-random manner and the animal asked to search for the numeral in question ( 5 , in this example). As the child's mastery improved, the numeral span was expanded (e.g., a child who knew $1-10$ would be taught 11 - 15, and so forth).

Letter recognition and identification, building letter/sound correspondence, and distinguishing sounds were addressed in literacy instruction. The activities progressed in difficulty from using the alphabet chart to distinguishing different sounds. This session began with a warm up activity that focused on letter recognition and identification. Each child was given an alphabet chart and was instructed to have its animal read each letter. Then focus letters (both upper and lowercase) for that day were placed in front of the child and they were asked to have their animal point to a specific letter (e.g., "Show me the M."). Or, they might be shown one focus letter (e.g., Mm) and their animal asked what letter it was (e.g., "Tell me what letter this is?"). This process was repeated with children who did not know the letter name by restating the name of the letter and asking the children to repeat the letter name. Again, each child was instructed in turn while the other children watched and learned vicariously. Because it was always 
the toy animal which was correct or, if in error, got to have a second chance, instruction proceeded smoothly and enjoyably. The next task in the hierarchy was designed to help children connect to prior knowledge. Children's animals were asked whose name in the class began with the focus letter (e.g., Megan, Michael, Mason, etc.). The sound of the focus letter was emphasized in saying the children's names. The children were then asked what other words they know that begin with the focus letter (e.g., Monday, monkey, money, mommy, etc.).

Letter bags were utilized to help children build letter/sound correspondence and distinguish between different beginning sounds. Some objects or pictures of objects that began with the focus letter as well as some objects or pictures of objects that didn't begin with the focus letter were placed in front of the children. Referring to each object, children's toy animals were asked if it began with the focus letter (e.g., "What is this?" "Does monkey begin with an M sound?" "Does cat begin with the $\mathrm{M}$ sound?"). If more support was needed, the beginning sound was emphasized and the child asked "Does monkey begin with an $\mathrm{M}$ sound like mommy?" If even more assistance was needed, the beginning sound would be emphasized with corresponding objects. Only the objects or pictures of objects that corresponded to the focus letter were used, and the children were asked to say each word together with the teacher. The same letter bags were used to teach children distinguish between different beginning sounds. For this task, two or more previously learned letters (e.g., $\mathrm{C}$ and F) were included in each session. Objects or pictures of objects that began with these letters were put in front of the children. Children were asked to sort the objects or pictures by placing the ones whose names began with $\mathrm{C}$ in one bag and the ones that began with $\mathrm{F}$ in the other bag. If more support was needed, the beginning sound was emphasized and the children were asked, "Does cat begin like can or does cat begin like fan?" In addition, note cards were used to teach children phonics with a group of words with the same root (e.g., - air family consisted of cards such as chair, flair, hair, lair, pair, and stair). At the end of the literacy instruction, previous focus letters would review with children who did not identify them correctly.

For social studies instruction, a variety of topics that highlighted historical events and people, geography, and civics were featured in activities with play doh, crayons, and papers with various representations of these topics. Frequent interactions between the children and their toy animals were used to facilitate meaningful engagement.

In late April-early May, children in all four conditions were post tested, in a counterbalanced order, with the patterning, mathematics, early literacy, and executive function tests.

\section{Results}

\subsection{Analyses of Means}

Inasmuch as the pretest stratification and randomization procedure had equalized the children initial scores on patterning, literacy, and mathematics, F ( 3 , 
168) ranged from $0.33-0.61, p>0.60$, analyses were based on post-test scores.

The children who were taught patterning scored better on the 30 -item patterning test than the children who received any other form of instruction. However, ANOVA with planned comparisons showed that the only significant differences were between those who were taught patterning and those who were taught mathematics $(p<0.036)$ or social studies $(p<0.029)$. Children who were taught literacy made lower scores than those taught patterning, but not significantly lower $(p=0.131)$. The patterning group averaged $71 \%$ correct on the patterning measure; the other groups averaged $62 \%-65 \%$.

There were no differences approaching significance on any other measure (see Table 1). Hence the scores of all of the children were combined for additional analyses.

\subsection{Correlational Analyses}

Correlational analyses showed that nearly all measures were significantly correlated.

Table 1. Descriptive statistics for each instructional condition.

\begin{tabular}{|c|c|c|c|c|c|c|c|c|}
\hline \multirow{3}{*}{ Dependent Variable } & \multicolumn{8}{|c|}{ Instructional Condition } \\
\hline & $\mathbf{M}$ & SD & $\mathbf{M}$ & $\mathrm{SD}$ & $\mathbf{M}$ & $\mathrm{SD}$ & $\mathbf{M}$ & SD \\
\hline & \multicolumn{2}{|c|}{ Patterning } & \multicolumn{2}{|c|}{ Mathematics } & \multicolumn{2}{|c|}{ Literacy } & \multicolumn{2}{|c|}{ Social Studies } \\
\hline Patterning Test & 17.02 & 5.75 & 14.94 & 5.62 & 15.71 & 4.63 & 14.83 & 5.89 \\
\hline Math Computation & 13.86 & 5.37 & 15.44 & 5.63 & 14.87 & 5.41 & 15.04 & 5.01 \\
\hline Math Concepts & 18.75 & 3.72 & 19.9 & 2.55 & 19.38 & 3.63 & 18.96 & 4.03 \\
\hline TOWRE Phonemics & 7.7 & 9.73 & 8.25 & 8.12 & 7.29 & 5.59 & 5.98 & 7.15 \\
\hline TOWRE Sight Words & 15.77 & 15.33 & 16.27 & 13.72 & 18.84 & 12.91 & 16 & 14.46 \\
\hline DIBELS Initial Sounds & 11.82 & 3.43 & 12.13 & 3.63 & 12.69 & 3.55 & 12.71 & 7.68 \\
\hline DIBELS Letters Named & 39.95 & 20.97 & 38.85 & 15 & 38.42 & 17.04 & 42.9 & 17.36 \\
\hline DIBELS Letters Attempted & 42.7 & 20.29 & 41.02 & 14.53 & 41.71 & 15.81 & 45.77 & 16.19 \\
\hline MCCST Accuracy & 9.52 & 4.42 & 9.38 & 4.71 & 10.8 & 4.42 & 10.1 & 4.76 \\
\hline MCCST Justifications & 4.5 & 2.73 & 4.65 & 2.5 & 4.49 & 4.23 & 4.23 & 2.62 \\
\hline MCCST Time & 220.36 & 147.66 & 198.32 & 67.72 & 216.65 & 82.6 & 194.37 & 63.64 \\
\hline MCCST Composite & 8.34 & 6.09 & 7.5 & 4.31 & 8.31 & 4.85 & 7.99 & 4.7 \\
\hline Day-Night Correct & 14.2 & 2.75 & 14.06 & 2.94 & 13.84 & 3.94 & 13.45 & 4.23 \\
\hline Day-Night Time & 73 & 29.14 & 66.1 & 33.77 & 70.55 & 32.8 & 64.37 & 20.7 \\
\hline Day-Night Ratio & 0.16 & 0.16 & 0.47 & 1.14 & 0.23 & 0.11 & 0.34 & 0.73 \\
\hline Digit Span Memory & 2 & 2.09 & 2.58 & 2.14 & 2.53 & 2.36 & 2.44 & 2.27 \\
\hline HTKS inhibition & 44.2 & 9.78 & 40.55 & 14.53 & 44.69 & 15.77 & 43.57 & 15.04 \\
\hline
\end{tabular}

Note. Post test means and standard deviations are given for each instructional condition. 
The correlation coefficient is the effect size. Cohen's (1992) rubric suggests that correlations between 0.10 and 0.30 are small, those between 0.30 and 0.50 are medium, and those over 0.50 are large.

\subsubsection{Correlations with Patterning}

The highest correlations for accuracy on the patterning test were with the two math measures, Concepts $\mathrm{r}(183)=0.420, p<0.001$ and Computation, $\mathrm{r}(183)=$ $0.438, p<0.001$. There were also correlations with the early literacy measures, ranging from $\mathrm{r}(182)=0.205, p<0.003$ to $\mathrm{r}(182)=0.325, p<0.003$.

Patterning correlated significantly with both the HTKS inhibition measure, $\mathrm{r}$ $(58)=0.360, p<0.002$, and the children's accuracy on another inhibition measure, the Day-Night test, $\mathrm{r}(183)=0.316, p<0.001$. The correlations between patterning and the time the children took to complete the Day-Night measure and the ration of accuracy to time were small and insignificant, $\mathrm{r}(183)=0.020, p>$ 0.05 and $\mathrm{r}(183)=-0.076, p>0.05$, respectively.

There also significant correlations between patterning accuracy and scores on both working memory measures. For patterning and the Backwards Digit Span, $\mathrm{r}(158)=0.374, p<0.001$; for the patterning and the Corsi Blocks test, $\mathrm{r}(58)=$ $0.389, p<0.001$.

Accuracy on the patterning test was significantly correlated with accuracy on the MCCST cognitive flexibility measure, $\mathrm{r}(183)=0.150, p<0.05$, with children's justifications for their answers, $\mathrm{r}(183)=0.251, p<0.001$, and with a composite measure, $\mathrm{r}(183)=0.255, p<0.001$. The time it took to complete the MCCST was not correlated with patterning, $\mathrm{r}(183)=-0.014, p>0.05$.

\subsubsection{Correlations between Different EF Measures of the Same Constructs}

HTKS inhibition scores did not correlate significantly with accuracy on the Day-Night inhibition test, $\mathrm{r}(58)=0.158, p>0.05$, nor with the time it took the children to complete this test, $\mathrm{r}(58)=0.016, p>0.05$. However, the ratio of the number of items they answered correctly divided by time it took them did correlate significantly with accuracy on the HTKS, $\mathrm{r}(58)=-0.418, p<0.001$. That is to say that children who were able to answer in fewer seconds while getting many answers correct, producing a relatively small ratio, made high scores on the HTKS, showing good inhibition ability and producing a negative $r$.

The correlation between the two working memory measures, Backwards Digit Span and Corsi Blocks, was significant, $\mathrm{r}(58)=0.26, p<0.022$.

The composite MCCST measure of cognitive flexibility, a ratio of sorting accuracy plus scores for justifications divided by the time it took to complete the MCCST, and then multiplied by 100 , did correlate with both accuracy on the MCCST, $\mathrm{r}(183)=0.308, p<0.001$, and the time it took children to complete this measure, $\mathrm{r}(183)=-0.493, p<0.001$. It also correlated with their ability to justify their answers, $\mathrm{r}(183)=0.594, p<0.001$. Justifications also correlated with accuracy, $\mathrm{r}(183)=0.270, \mathrm{p}=0.001$, but not with time $\mathrm{r}(183)=-0.105, p>0.05$. 


\subsubsection{Correlations between EF Measures of Different Constructs}

Corsi Blocks working memory scores correlated with HTKS inhibition scores, $r$ $(58)=0.632 . p<0.001$ - the highest correlation found in this study. The Digit Span working memory scores also correlated with HTKS inhibition scores, $\mathrm{r}$ (58) $=0.304, p<0.009$, but the correlation was only half as large for this working memory measure.

Corsi Blocks scores also correlated significantly with accuracy on the Day-Night inhibition measure, $\mathrm{r}(58)=0.343, p<0.031$, and with the ratio of accuracy to time, $\mathrm{r}(58)=0.331, p<0.006$. Digit Span working memory scores also correlated significantly with Day-Night accuracy, although the correlation was much smaller, $\mathrm{r}(183)=0.134, p<0.035$. There was a small correlation between Digit Span scores and the time the children took on the Day-Night measure, $\mathrm{r}(183)=-0.162, p<0.014$, but not with the ratio of time to accuracy.

Corsi Blocks working memory scores correlated with three MCCST cognitive flexibility measures: justifications, $\mathrm{r}(58)=0.389, p<0.001$, time $\mathrm{r}(58)=-0.230$, $p<0.038$, and the composite scores, $\mathrm{r}(58)=0.550, p<0.001$. Digit Span working memory scores correlated with MCCST justifications, $\mathrm{r}(183)=0.267, p<$ 0.001 , and composites, $\mathrm{r}(183)=0.202, p<0.003$, but not the total time children spent on the MCCST.

HTKS inhibition scores also correlated with MCCST justifications, $\mathrm{r}(58)=$ $0.453, p<0.001$, and composites, $\mathrm{r}(58)=0.480, p<0.001$, but not with the total time children spent on the MCCST. Day-Night inhibition correlations were also significant for the same MCCST measures, $\mathrm{r}(183)=0.224, p<0.001$ for justifications, and $\mathrm{r}(183)=0.146, p<0.024$ for composites, but much smaller.

\subsubsection{Correlations between EF Measures and Mathematics}

The correlations between the HTKS inhibition measure and both mathematics measures were close to Cohen's (1992) criterion (0.50) for strong effects; $r$ (183) $=0.485, p<0.001$ for Math Concepts, and $\mathrm{r}(183)=0.530, p<0.001$ for Math Computations. On the Day-Night inhibition test, correlations for accuracy, $r$ $(183)=0.373, p<0.001$, and time expended $\mathrm{r}(183)=-0.227, p<0.001$, represented medium and small effects, respectively.

The Corsi blocks measure of working memory was correlated with both mathematics measures, $\mathrm{r}(58)=0.442, p<0.001$ for Math Concepts and $\mathrm{r}(58)=$ 0.548, $p<0.001$ for Mathematics Computations. Backwards Digit Span measures of working memory also correlated with both Mathematics Concepts, $r(183)=$ $0.375, p<0.001$, and Computations, $\mathrm{r}(183)=0.436, p<0.001$.

The time it took children to perform the MCCST card sort was correlated with their Math Computation scores, $\mathrm{r}(183)=-0.200, p<0.003$, but the accuracy of their sorting was not. Neither of these cognitive flexibility measures were correlated with Math Concepts.

On the other hand, the children's ability to justify the sorting they had done did correlate with both Math Concepts scores $\mathrm{r}(183)=0.352, p<0.001$, and Math Computation scores, $\mathrm{r}(183)=0.359, p<0.001$. Likewise, composite scores 
for this cognitive flexibility measure, Sorting Accuracy plus Justification divided by Time and multiplied by $100 \%$, correlated with both Math Concepts, $\mathrm{r}(183)=$ $0.269, p<0.001$, and Math Computation scores, $\mathrm{r}(183)=0.382, p<0.001$.

\subsubsection{Correlations between EF Measures and Early Literacy}

HTKS inhibition scores correlated significantly $(0.282-0.442)$ with all of the early literacy measures except the DIBELS ratio of letters named divided by letters attempted. Accuracy on the Day-Night inhibition test correlated significantly with all of the early literacy measures except the DIBELS initial sounds scale. The significant correlations ranged from 0.217 to 0.317 . The Day-Night time measure correlated with all DIBELS and TOWRE scores except for DIBELS correct initial sounds per minute. However, the Day-Night ratio of correct answers per minute did not correlate significantly with any early literacy measures (see Table 2).

\subsubsection{Correlations between Achievement Measures}

The correlation between the two mathematics scales was $\mathrm{r}(183)=0.653, p<$ 0.001 ; the correlation between the two TOWRE early literacy scales was $\mathrm{r}(183)=$ $0.810, p<0.001$. The highest correlation between the five DIBELS scales was that for letters named versus letters attempted, $\mathrm{r}(182)=0.973, \mathrm{p}<0.001$. The lowest

Table 2. Correlations of patterning and inhibition with early literacy scales.

\begin{tabular}{|c|c|c|c|c|c|c|c|}
\hline \multicolumn{8}{|c|}{ Correlations of Patterning with Early Literacy Scales } \\
\hline & $\begin{array}{l}\text { TOWRE } \\
\text { Words }\end{array}$ & $\begin{array}{c}\text { TOWRE } \\
\text { Phonemes }\end{array}$ & DIBELS Correct & $\begin{array}{l}\text { DIBELS } 1^{\text {st }} \\
\text { Sounds }\end{array}$ & $\begin{array}{l}\text { DIBELS Letters } \\
\text { Named }\end{array}$ & $\begin{array}{l}\text { DIBELS Letters } \\
\text { Attempted }\end{array}$ & $\begin{array}{c}\text { DIBELS } \\
\text { Ratio }\end{array}$ \\
\hline $\mathbf{r}$ & 0.31 & 0.25 & 0.3 & 0.33 & 0.23 & 0.22 & 0.21 \\
\hline $\mathrm{p}$ & 0.001 & 0.001 & 0.001 & 0.001 & 0.001 & 0.002 & 0.003 \\
\hline \multicolumn{8}{|c|}{ Correlations of HTKS Inhibition Scale with Early Literacy Scales } \\
\hline & TOWRE Words & $\begin{array}{c}\text { TOWRE } \\
\text { Phonemes }\end{array}$ & DIBELS Correct & $\begin{array}{l}\text { DIBELS } 1^{\text {st }} \\
\text { Sounds }\end{array}$ & $\begin{array}{l}\text { DIBELS Letters } \\
\text { Named }\end{array}$ & $\begin{array}{l}\text { DIBELS Letters } \\
\text { Attempted }\end{array}$ & DIBELS Ratio \\
\hline $\mathbf{r}$ & 0.42 & 0.32 & 0.33 & 0.43 & 0.36 & 0.38 & 0.17 \\
\hline $\mathrm{p}$ & 0.001 & 0.001 & 0.001 & 0.001 & 0.001 & 0.001 & $>0.05$ \\
\hline \multicolumn{8}{|c|}{ Correlations of Day-Night Inhibition Accuracy Scale with Early Literacy Scales } \\
\hline & TOWRE Words & $\begin{array}{c}\text { TOWRE } \\
\text { Phonemes }\end{array}$ & DIBELS Correct & $\begin{array}{l}\text { DIBELS } 1^{\text {st }} \\
\text { Sounds }\end{array}$ & $\begin{array}{l}\text { DIBELS Letters } \\
\text { Named }\end{array}$ & $\begin{array}{l}\text { DIBELS Letters } \\
\text { Attempted }\end{array}$ & DIBELS Ratio \\
\hline $\mathbf{r}$ & 0.23 & 0.22 & 0.24 & 0.09 & 0.3 & 0.28 & 0.32 \\
\hline $\mathrm{p}$ & 0.001 & 0.001 & 0.001 & $>0.05$ & 0.001 & 0.001 & 0.001 \\
\hline \multicolumn{8}{|c|}{ Correlations of Day-Night Inhibition Time Scale with Early Literacy Scales } \\
\hline & TOWRE Words & $\begin{array}{c}\text { TOWRE } \\
\text { Phonemes }\end{array}$ & DIBELS Correct & $\begin{array}{l}\text { DIBELS } 1^{\text {st }} \\
\text { Sounds }\end{array}$ & $\begin{array}{l}\text { DIBELS Letters } \\
\text { Named }\end{array}$ & $\begin{array}{l}\text { DIBELS Letters } \\
\text { Attempted }\end{array}$ & DIBELS Ratio \\
\hline $\mathbf{r}$ & -0.21 & -0.17 & -0.1 & -0.13 & -0.24 & -0.24 & -0.18 \\
\hline $\mathrm{p}$ & 0.002 & 0.012 & $>0.05$ & 0.04 & 0.001 & 0.001 & 0.009 \\
\hline
\end{tabular}

Note. Values of $\mathrm{p}$ are two-tailed. 
was that between initial sound fluency and the ratio of letters named correctly to letters attempted, $\mathrm{r}(182)=0.279, p<0.001$.

All of the DIBELS early literacy measures correlated significantly with both TOWRE early literacy measures and both mathematics measures. Correlations of the DIBELS scales with the TOWRE scales ranged from 0.312 to 0.575 . All $\mathrm{p}$ values were $<0.001$ (see Table 3 ). Correlations of the DIBELS scales with Mathematics Concepts were in the 0.30 s. Correlations with Mathematics Computations ranged from a low of 0.349 to a high of 0.547 (see Table 3 ).

\subsubsection{Exploratory Factor Analysis}

A factor analysis of these data, $X^{2}(151)=403.99, p>0.001$, revealed two factors (see Table 4). One factor consisted of the early literacy measures and the two mathematics scales (Concepts and Computations). The time it took children to complete the Day-Night inhibition measure also loaded on this factor. (Note that the loading is negative, as long times indicate poor performance.) The mathematics Computation measure also loaded on a second factor, which included patterning scores, both HTKS inhibition measures, two MCCST cognitive flexibility measures, and the Corsi blocks working memory measure. Thus, the first factor is primarily an achievement measure, both in terms of early literacy and mathematics, whereas the second factor is primarily a measure of the children's

Table 3. Correlations with literacy achievement measures.

\begin{tabular}{|c|c|c|c|c|c|c|c|}
\hline \multicolumn{8}{|c|}{ Correlations of Mathematics Concepts Scale with Early Literacy Scales } \\
\hline & TOWRE Words & $\begin{array}{c}\text { TOWRE } \\
\text { Phonemes }\end{array}$ & DIBELS Correct & $\begin{array}{l}\text { DIBELS } 1^{\text {st }} \\
\text { Sounds }\end{array}$ & $\begin{array}{c}\text { DIBELS Letters } \\
\text { Named }\end{array}$ & $\begin{array}{l}\text { DIBELS Letters } \\
\text { Attempted }\end{array}$ & DIBELS Ratio \\
\hline $\mathbf{r}$ & 0.38 & 0.31 & 0.33 & 0.39 & 0.39 & 0.37 & 0.37 \\
\hline $\mathrm{p}$ & 0.001 & 0.001 & 0.001 & 0.001 & 0.001 & 0.001 & 0.001 \\
\hline \multicolumn{8}{|c|}{ Correlations of Mathematics Computations Scale with Early Literacy Scales } \\
\hline & TOWRE Words & $\begin{array}{c}\text { TOWRE } \\
\text { Phonemes }\end{array}$ & DIBELS Correct & $\begin{array}{l}\text { DIBELS } 1^{\text {st }} \\
\text { Sounds }\end{array}$ & $\begin{array}{c}\text { DIBELS Letters } \\
\text { Named }\end{array}$ & $\begin{array}{l}\text { DIBELS Letters } \\
\text { Attempted }\end{array}$ & DIBELS Ratio \\
\hline $\mathbf{r}$ & 0.55 & 0.45 & 0.42 & 0.41 & 0.47 & 0.46 & 0.35 \\
\hline $\mathrm{p}$ & 0.001 & 0.001 & 0.001 & 0.001 & 0.001 & 0.001 & 0.001 \\
\hline \multicolumn{8}{|c|}{ Correlations of TOWRE Words Scale with DIBELS Scales } \\
\hline & DIBELS Correct & $\begin{array}{l}\text { DIBELS } 1^{\text {st }} \\
\text { Sounds }\end{array}$ & $\begin{array}{l}\text { DIBELS Letters } \\
\text { Named }\end{array}$ & $\begin{array}{l}\text { DIBELS Letters } \\
\text { Attempted }\end{array}$ & DIBELS Ratio & & \\
\hline $\mathbf{r}$ & 0.53 & 0.4 & 0.57 & 0.58 & 0.31 & & \\
\hline $\mathbf{p}$ & 0.001 & 0.001 & 0.001 & 0.001 & 0.001 & & \\
\hline \multicolumn{8}{|c|}{ Correlations of TOWRE Phonemics Scale with DIBELS Scales } \\
\hline & DIBELS Correct & $\begin{array}{l}\text { DIBELS } 1^{\text {st }} \\
\text { Sounds }\end{array}$ & $\begin{array}{l}\text { DIBELS Letters } \\
\text { Named }\end{array}$ & $\begin{array}{l}\text { DIBELS Letters } \\
\text { Attempted }\end{array}$ & DIBELS Ratio & & \\
\hline $\mathbf{r}$ & 0.45 & 0.33 & 0.55 & 0.54 & 0.34 & & \\
\hline $\mathrm{p}$ & 0.001 & 0.001 & 0.001 & 0.001 & 0.001 & & \\
\hline
\end{tabular}

Note. Values of p are two-tailed. 
Table 4. Exploratory factor analysis.

\begin{tabular}{|c|c|c|c|}
\hline \multicolumn{4}{|c|}{ Component Loadings } \\
\hline & $\mathrm{RC} 1$ & $\mathrm{RC} 2$ & Uniqueness \\
\hline KTEA Concepts & 0.515 & & 0.458 \\
\hline KTEA Computation & 0.461 & 0.46 & 0.434 \\
\hline TOWRE Words & 0.614 & & 0.451 \\
\hline TOWRE Phonemes & 0.632 & & 0.571 \\
\hline \multicolumn{4}{|l|}{ DIBELS } \\
\hline $1^{\text {st }}$ Sounds & & & 0.738 \\
\hline Correct & 0.684 & & 0.537 \\
\hline Letters Named & 0.988 & & 0.113 \\
\hline Letters Attempted & 0.91 & & 0.215 \\
\hline Ratio & 0.543 & & 0.737 \\
\hline Patterning & & 0.555 & 0.65 \\
\hline Digit Span Memory & & & 0.776 \\
\hline Corsi Memory & & 0.702 & 0.415 \\
\hline Day-Night Correct & & & 0.859 \\
\hline Day-Night Time & -0.439 & & 0.779 \\
\hline HTKS Inhibition & & 0.629 & 0.43 \\
\hline MCCST Accuracy & & & 0.88 \\
\hline MCCST Justification & & 0.478 & 0.599 \\
\hline MCCST Time & & & 0.976 \\
\hline MCCST Ratio & & 0.635 & 0.514 \\
\hline
\end{tabular}

Note. Loadings on each of two factors are given. Two scales loaded on both.

current thinking abilities.

\section{Discussion}

\subsection{Major Findings}

Eight weeks of instruction of kindergartners on $\mathrm{AB}, \mathrm{ABB}$, and more complex patterns had a significant but small effect on children's patterning as measured by the tests used here. There was no indication that this patterning instruction had an effect on mathematics similar to that reported by Kidd et al. (2013) for first graders nor on both mathematics and literacy as was reported by Kidd et al. (2014) and Pasnak et al. (2015).

The study does provide a very extensive description of relations between patterning, early literacy, mathematics, and EF. The children's patterning scores were correlated with mathematics and literacy scores, whether or not they had received instruction in patterning, literacy, mathematics, or social studies. Coefficients of determination indicate that at the end of kindergarten about $16 \%$ of 
the differences in their mathematics achievement was linearly related to difference in their patterning ability, as measured here. This relation is in line with the longitudinal study by Rittle-Johnson et al. (2016), the findings of Miller et al., (2016) for preschoolers, and the findings of Kidd et al. (2013), Kidd et al. (2014), MacKay and De Smedt (2019), and Pasnak et al. (2015) for older children. The coefficients of determination for early literacy were smaller (4\% - 9\%). Hence, young children's patterning is primarily related to mathematics, as frequently theorized, even if it is also related to literacy.

Factor analysis of the present data offers a partial explanation, in that EF measures and patterning loaded on one factor, which might be termed cognitive ability, and the achievement measures loaded on the other, with one mathematics measure (computation) loading on both. It is interesting that it is computation, which MacKay and De Smedt (2019) showed to be related to understanding number, time, and letter patterns for seven-year-old, that loaded on the same factor as patterning in this research. Mathematics Concepts, which might be considered a more likely candidate, did not, at least as measured here.

The factor analysis and the correlations with mathematics obtained indicate that patterning shares with other cognitive abilities a relation to mathematics performance. The correlation of patterning with mathematics computations and concepts were medium effects. The correlations of the various EF measures with mathematics concepts and computation ranged more broadly, depending on how they were measured, but those which were significant were generally medium effects. The expressions of EF do share a common variance (Miyake et al., 2000) but are also separable. The same appears to be true for the EF and patterning. The highest correlations for kindergartners of patterning with these EF as assessed in this experiment were with working memory and inhibition; correlations with cognitive flexibility were lower. There is a commonality, but patterning appears to be a distinct ability.

In this context, it is worth noting that the correlation between the two working memory measures used here was small. Although it was statistically significant, the coefficient of determination indicates that less than $7 \%$ of the differences between children's scores on one measure are predictable from their differences on the other measure of the same EF.

One noteworthy result of this research is that both inhibition measures were shown related to be patterning. Collins and Laski (2015) had previously found a relation of HTKS inhibition scores to patterning for preschoolers, but Miller et al. (2015) did not find such a relation with the Day-Night inhibition test for preschoolers and neither did Schmerold et al. (2017) or Bock et al. (2018) for first graders. The difficulty appears to lie in the difficulty of the measures. The Day-Night test is too easy for first graders, who made scores near ceiling (Bock et al., 2018; Schmerold et al., 2017); the HTKS was too difficult for the preschoolers of Mohtasham, Patterson, Vennergrund, Chen, and Pasnak (2018), who abandoned it after children complained that the HTKS "game" was too difficult. 
Different levels of inhibitory ability in samples from different populations appear to combine with differences in the demands of the measures to produce differences in the relations observed between inhibition and patterning.

\subsection{Limitations}

There are several possible reasons for the difference in outcomes of patterning instruction here and that reported for first graders (six-year-olds) by Kidd et al. (2013), Kidd et al. (2014), and Pasnak et al. (2015). One is the difference in samples. Pasnak, Hansbarger, Dodson, Hart, and Blaha (1996) reported that the same cognitive intervention carried out simultaneously in two different schools had a marked effect on achievement in one school but not in the other. The difference between the kindergartners and first graders may well have been the cause of the difference in results.

These young children in the present sample were very variable, producing low power in the analysis. At least 31 languages, some of them tribal dialects or unidentified languages were spoken in the homes of these children. Although recognizing patterns might be culture free, response to literacy and mathematics measures given in English was much more difficult for some children than others. The resulting variability in communication added to variability in the measures of patterning and achievement.

There is also the fact that the patterning and achievement tests used here are not the same as those used by Kidd et al. (2013), nor, except for the TOWRE, with those used by Kidd et al. (2014), and Pasnak et al. (2015). We note that Kidd et al. (2013) did not find effects on three Woodcock-Johnson literacy scales, but Kidd et al. (2014) and Pasnak et al. (2015) did find them on three other literacy tests. Literacy and mathematics achievement at kindergarten age is hard to measure, and different scales might have produced different results.

Another consideration is that the multilayered approval system in the school district delayed the beginning of the patterning instruction until late November. Shriver et al. (2017) argued that to have an effect on achievement, any cognitive intervention has to be begun early in the school year, so that the children's improved cognitive abilities are in place in time to improve the children's understanding of the lessons the teacher and curriculum deliver to the whole class. Better understanding, produced by improvement in patterning by the children receiving the experimental patterning, could lead to more profit for them from the literacy and mathematics lessons delivered to all of the children in each classroom. However, that would only be the case after their understanding of patterns had been substantially advanced. If that advancement did not come early in the school year, the opportunity to apply their enhanced reasoning abilities to classroom lessons in literacy and mathematics would be lost, or at least reduced. That may be what happened here.

Perhaps more important is that the teachers in the cooperating school system did teach their whole classes $\mathrm{AB}, \mathrm{ABB}, \mathrm{BBA}, \mathrm{AABB}$, and $\mathrm{ABC}$ patterns. The ex- 
perimental instruction overlapped this. It is apparent that extension of patterning instruction to symmetric and random repeating patterns did not affect achievement as measured here.

The diversity of measures of working memory, cognitive flexibility, and inhibition used by different researchers add to the effects of sample differences in determining how they relate to patterning. Even when the same test is used (the WISC-R Backwards Digit Span measure of working memory), there are at least three different ways of calculating a child's score. The most direct was used here, but other calculations and administrations are certainly defensible.

A related issue is that relations between EF and both literacy (Cartwright, 2002) and patterning (Mohtasham et al., 2018) change rapidly when children are young. Mohtasham et al. (2018) found that relations existing early in the year disappeared over a period of six months. The probability of replicability in demonstrations of relations between patterning and EF is likely to be low.

It is also apparent that two months of thrice weekly indirect instruction of trios of children from this sample did not produce gains in literacy or mathematics as measured here. This may reflect the nature and variability of the children in the sample, or the methods of instruction, or the choice of achievement tests. Whichever of these problems combined to produce the lack of improvement from direct instruction in the measures of early literacy and mathematics may also have prevented the small (six to nine per cent) gain in patterning ability for the experimental group from having a detectable effect.

\section{Conclusion}

This experiment appears to show that instruction of kindergartners on two kinds of repeating patterns, symmetric patterns, and two kinds of patterns belonging to a class MacKay and De Smedt (2019) termed sequential patterns did not produce significant gains in literacy or mathematics. The extent to which a child masters such patterns is, however, linearly related to literacy and especially, mathematics scores. Relations between each EF measure, patterning, and each achievement measure were identified; these are generally medium effects $(r=$ 0.30 - 0.50), although smaller effects were also observed. Factor analysis showed that patterning and EF measures, together with mathematics computations, form a factor that expresses thinking abilities, while all of the achievement measures form another. Given the demonstration that relations between this form of patterning and achievement exist, more propitious timing and methods of patterning instruction, or different measures of achievement, or a sample of children with fewer language difficulties might produce a better result.

\section{Support Information}

The research reported here was supported by the Institute of Education Sciences, U.S. Department of Education, through Grant 305A170114 to George Mason University. The opinions expressed are those of the authors and do not represent 
views of the Institute or the U.S. Department of Education.

\section{Conflicts of Interest}

The authors declare no conflicts of interest regarding the publication of this paper.

\section{References}

Bjorklund, C., \& Pramling, N. (2014). Pattern Discernment and Pseudo-Conceptual Development in Early Childhood Mathematics Education. International Journal of Early Years Education, 22, 89-104. https://doi.org/10.1080/09669760.2013.809657

Bock, A. B., Cartwright, K. B., McKnight, P. E., Patterson, A. B., Shriver, A. S., Leaf, B. M., Mohtasham, M. K., Vennergrund, K. C., \& Pasnak, R. (2018). Patterning, Reading, and Executive Functions. Frontiers in Psychology, 9, 1802. https://doi.org/10.3389/fpsyg.2018.01802

Cartwright, K. B. (2002). Cognitive Development and Reading: The Relation of Reading-Specific Multiple Classification Skill to Reading Comprehension in Elementary School Children. Journal of Educational Psychology, 94, 56-63. https://doi.org/10.1037/0022-0663.94.1.56

Chasiotis, A., Kiessling, F., Hofer, J., \& Campos, D. (2006). Theory of Mind and Inhibitory Control in Three Cultures: Conflict Inhibition Predicts False Belief Understanding in Germany, Costa Rica and Cameroon. International Journal of Behavioral Development, 30, 249-260. https://doi.org/10.1177/0165025406066759

Clements, D. H., \& Sarama, J. (2011). Early Childhood Mathematics Intervention. Science, 333, 968-970. https://doi.org/10.1126/science.1204537

Cohen, J. (1992). A Power Primer. Psychological Bulletin, 112, 155-159.

Collins, M. A., \& Laski, E. V. (2015). Preschoolers' Strategies for Solving Visual Pattern Tasks. Early Childhood Research Quarterly, 32, 204-214. https://doi.org/10.1016/j.ecresq.2015.04.004

Connolly, A. J. (2007). KeyMath-3 Diagnostic Assessment: Manual Forms A and B. Minneapolis, $\mathrm{MN}$ : Pearson.

Dubon, L. P., \& Shafer, K. G. (2010). Teaching Children Mathematics. National Council of Teachers of Mathematics, 16, 325-329.

Economopoulos, K. (1998). What Comes Next? The Mathematics of Pattern in Kindergarten. Teaching Children Mathematics, 5, 230-234.

Ferrara, F., \& Sinclair, N. (2016). An Early Algebra Approach to Pattern Generalization: Actualizing the Virtual through Words, Gestures and Toilet Paper. Education Study Mathematics, 92, 1-19. https://doi.org/10.1007/s10649-015-9674-3

Fyfe, E. R., Evans, J. L., Matz, L. E., Hunt, K. M., \& Alibali, M. W. (2017). Relations between Patterning Skill and Differing Aspects of Early Mathematics Knowledge. Cognitive Development, 44, 1-11. https://doi.org/10.1016/j.cogdev.2017.07.003

Fyfe, E. R., McNeil, N. M., \& Rittle-Johnson, B. (2015). Easy as ABCABC: Abstract Language Facilitates Performance on a Concrete Patterning Task. Child Development, 86, 927-935. https://doi.org/10.1111/cdev.12331

Good, R. H., Kaminski, R. A., Shinn, M., Bratten, J., Shinn, M., Laimon, L., Smith, S., \& Flindt, N. (2004). Technical Adequacy and Decision Making Utility of DIBELS (Technical Report No. 7). Eugene, OR: University of Oregon.

Kaminski, R. A., \& Good, R. H. (1996). Toward a Technology for Assessing Basic Early 
Literacy Skills. School Psychology Review, 25, 215-227.

Kaminski, R. A., \& Good, R. H. (1998). Assessing Early Literacy Skills in a Problem Solving Model: Dynamic Indicators of Basic Early Literacy Skills. In M. R. Shinn (Ed.), Advanced Applications of Curriculum-Based Measurement (pp. 113-142). New York: Guilford.

Kaufman, A. S., \& Kaufman, N. L. (2014). Kaufman Test of Educational Achievement (3rd ed.). Bloomington, MN: Pearson.

Kessels, R. P. C., van Zandvoort, M. J. E., Postma, A., Kappelle, L. J., \& de Haan, E. H. F. (2000). The Corsi Block-Tapping Task: Standardization and Normative Data. Applied Neuropsychology, 7, 252-258. https://doi.org/10.1207/S15324826AN0704_8

Kidd, J. K., Carlson, A., Gadzichowski, K. M., Boyer, C. E., Gallington, D. A., \& Pasnak, R. (2013). Effects of Patterning Instruction on the Academic Achievement of 1st-Grade Children. Journal of Research on Childhood Education, 27, 224-238. https://doi.org/10.1080/02568543.2013.766664

Kidd, J. K., Pasnak, R., Gadzichowski, K. M., Gallington, D. A., McKnight, P., Boyer, C. E., \& Carlson, A. (2014). Instructing First-Grade Children on Patterning Improves Reading and Mathematics. Early Childhood Development, 25, 134-151. https://doi.org/10.1080/10409289.2013.794448

Laimon, D. E. (1994). The Effects of a Home-Based and Center-Based Intervention on at Risk Preschool Children's Early Literacy Skills. Unpublished Doctoral Dissertation, Eugene, OR: University of Oregon.

MacKay, K. J., \& De Smedt, B. (2019). Patterning Counts: Individual Differences in Children's Calculation Are Uniquely Predicted by Sequence Patterning. Journal of EXperimental Child Psychology, 177, 152-165. https://doi.org/10.1016/j.jecp.2018.07.016

Marston, D., \& Magnusson, D. (1988). Curriculum-Based Measurement: District Level Implementation. In J. Graden, J. Zins, \& M. Curtis (Eds.), Alternative Educational Delivery Systems: Enhancing Instructional Options for All Students (pp. 137-172). Washington, DC: National Association of School Psychology.

McGarvey, L. M. (2012). What Is a Pattern? Criteria Used by Teachers and Young Children. Mathematical Thinking and Learning, 14,310-337. https://doi.org/10.1080/10986065.2012.717380

Miller, M. R., Rittle-Johnson, B., Loehr, A. M., \& Fyfe, E. R. (2015). The Influence of Relational Knowledge and Executive Function on Preschoolers' Repeating Pattern Knowledge. Journal of Cognition and Development, 17, 85-104. https://doi.org/10.1080/15248372.2015.1023307

Miyake, A., Friedman, N. P., Emerson, M. J., Witzki, A. H., Howerter, A., \& Wager, T. (2000). The Unity and Diversity of Executive Functions and Their Contributions to Complete "Frontal Lobe" Tasks: A Latent Variable Analysis. Cognitive Psychology, 41, 49-100. https://doi.org/10.1006/cogp.1999.0734

Mohtasham, M. K., Patterson, A. B., Vennergrund, K. C., Chen, E. S., \& Pasnak, R. (2018). Emotional Competence, Behavioural Patterning, and Executive Functions. Early Child Development and Care, 188, 1-10. https://doi.org/10.1080/03004430.2017.1403434

Mulligan, J. (2011). Towards Understanding the Origins of Children's Difficulties in Mathematics Learning. Australian Journal of Learning Difficulties, 16, 19-39. https://doi.org/10.1080/19404158.2011.563476

Mulligan, J. T., Mitchelmore, M. C., Kemp, C., Marston, J., \& Highfield, K. (2008). Encouraging Mathematical Thinking through Pattern and Structure: An Intervention in 
the First Year of Schooling. Macquarie University Research Online.

http://www.aamt.edu.au/

Mulligan, J., \& Mitchelmore, M. C. (2009). Awareness of Pattern and Structure in Early Mathematical Development. Mathematics Education Research Journal, 21, 33-49. https://doi.org/10.1007/BF03217544

Mulligan, J., Woolcott, G., Mitchelmore, M. C., \& Davis, B. (2017). Connecting Mathematics Learning through Spatial Reasoning. Mathematics Education Research, 30, 77-87. https://doi.org/10.1007/s13394-017-0210-x

Murray, J., Scott, H., Connolly, C., \& Wells, A. (2018). The Attention Training Technique Improves Children's Ability to Delay Gratification: A Controlled Comparison with Progressive Relaxation. Behaviour Research and Therapy, 104, 1-6. https://doi.org/10.1016/j.brat.2018.02.003

National Association for the Education of Young Children/National Council of Teachers of Mathematics Education (2002/2010). Early Childhood Mathematics Education: Promoting Good Beginnings. Washington DC: NYAEC.

National Council of Teachers of Mathematics (1993). Curriculum and Evaluation Standards for School Mathematics Addenda Series, Grades K-6. Reston, VA: NCTM.

National Council of Teachers of Mathematics (2000). Principles and Standards for School Mathematics. Reston, VA: NCTM.

National Council of Teachers of Mathematics (2006). Curriculum Focal Points for Prekindergarten through Grade 8 Mathematics: A Quest for Coherence. Reston, VA: NCTM.

Nguyen, T., Watts, T. W., Duncan, G. J., Clements, D. H., Sarama, J. S., Wolfe, C., \& Spitler, M. E. (2016). Which Preschool Mathematics Competences Are Most Predictive of Fifth Grade Achievement? Early Childhood Research Quarterly, 36, 550-560. https://doi.org/10.1016/j.ecresq.2016.02.003

Papic, M. (2007). Promoting Repeating Patterns with Young Children-More than Just Alternating Colors! Australian Primary Mathematics Classroom, 12, 8-12.

Papic, M. (2013). A Mathematics Intervention: The Case of 4-Year-Old Rylan and Hilda! Procedia-Social and Behavioral Sciences, 106, 92-101. https://doi.org/10.1016/j.sbspro.2013.12.012

Papic, M. (2015). An Early Mathematical Patterning Assessment: Identifying Young Australian Indigenous Children's Patterning Skills. Mathematics Education Research, 27, 519-534. https://doi.org/10.1007/s13394-015-0149-8

Papic, M., Mulligan, J. T., \& Mitchelmore, M. C. (2011). Assessing the Development of Preschoolers' Mathematical Patterning. Journal for Research in Mathematics Education, 42, 237-268. https://doi.org/10.5951/jresematheduc.42.3.0237

Pasnak, R., Hansbarger, A., Dodson, S., Hart, J., \& Blaha, J. (1996). Differential Results of Instruction at the Preoperational-Concrete Operational Transition. Psychology in the Schools, 33, 70-83.

Pasnak, R., Kidd, J. K., Gadzischowski, K. M., Gallington, D. A., Schmerold, K. L., \& West, H. (2015). Abstracting Sequences: Reasoning That Is a Key to Academic Achievement. The Journal of Genetic Psychology, 176, 171-193. https://doi.org/10.1080/00221325.2015.1024198

Ponitz, C. C., McClellan, M. M., Matthews, J. S., \& Morrison, F. J. (2009). A Structured Observation of Behavioural Self-Regulation and Its Contribution to Kindergarten Outcomes. Developmental Psychology, 45, 605-619.

https://doi.org/10.1037/a0015365 
Richardson, K., Berenson, S., \& Staley, K. (2009). Prospective Elementary Teachers' Use of Representation to Reason Algebraically. Journal of Mathematical Behavior, 28, 188-199. https://doi.org/10.1016/j.jmathb.2009.09.002

Rittle-Johnson, B., Fyfe, E. R., Hofer, K. G., \& Farran, D. C. (2016). Early Math Trajectories: Low-Income Children's Mathematics Knowledge from Ages 4 to 11. Child Development, 88, 1727-1742. https://doi.org/10.1111/cdev.12662

Rittle-Johnson, B., Fyfe, E. R., Loehr, A. M., \& Miller, M. R. (2015). Beyond Numeracy in Preschool: Adding Patterns to the Equation. Early Childhood Research Quarterly, 31, 101-112. https://doi.org/10.1016/j.ecresq.2015.01.005

Rittle-Johnson, B., Fyfe, E. R., McLean, L. E., \& McEldoon, K. L. (2013). Emerging Understanding of Patterning in 4-Year-Olds. Journal of Cognition and Development, 14, 376-396. https://doi.org/10.1080/15248372.2012.689897

Sarama, J., \& Clements, D. (2009a). Early Childhood Mathematics Education Research: Learning Trajectories for Young Children. New York: Routledge. https://doi.org/10.4324/9780203883785

Sarama, J., \& Clements, D. H. (2009b). Building Blocks and Cognitive Building Blocks: Playing to Know the World Mathematically. American Journal of Play, 1, 313-337. http://www.journalofplay.org/

Schmerold, K., Bock, A., Peterson, M., Leaf, B., Vennergrund, K., \& Pasnak, R. (2017). The Relations between Patterning, Executive Function, and Mathematics. The Journal of Psychology, 151, 207-228. https://doi.org/10.1080/00223980.2016.1252708

Shriver, A., Lauderdale, L., Yassa, M., Schroeder, E., Chen, E., Schabinger, E., Righi, M., \& Pasnak, R. (2017). The Effects of in-Class Tutoring of Kindergarteners on Patterning, Reading, or Mathematics. Journal of Education and Human Development, 6, 1-6. https://doi.org/10.15640/jehd.v6n1a1

Torgesen, J. K., Wagner, R. K., \& Rashotte, C. A. (2012). Test of Word Reading Efficiency Second Edition (TOWRE-2). Austin, TX: Pro-Ed.

Warren, E., \& Cooper, T. (2008). Generalizing the Pattern Rule for Visual Growth Patterns: Actions that Support 8 Years Olds' Thinking. Education Study Mathematics, 67, 171-185. https://doi.org/10.1007/s10649-007-9092-2

Warren, E., \& Miller, J. (2013). Young Australian Indigenous Students' Effective Engagement in Mathematics: The Role of Language, Patterns, and Structure. Mathematic Education Research Journal, 25, 151-171. https://doi.org/10.1007/s13394-013-0068-5

Wechsler, D. (2009). Wechsler Individual Achievement Test (3rd ed.). San Antonio, TX: NCS Pearson.

Wechsler, D. (2014). Wechsler Intelligence Scale for Children (5th ed.). Bloomington, MN: Pearson. 